\title{
Method of maximum likelihood estimation of optimal number of factors: An information criteria approach
}

\author{
Nwosu F. Dozie ${ }^{1}$, Onyeagu, Sidney I. ${ }^{2}$, Osuji, George A. ${ }^{2}$, Ekezie Dan Dan ${ }^{3}$ \\ ${ }^{1}$ Department of Mathematics and Statistics Federal Polytechnic Nekede, Owerri Imo State \\ ${ }^{2}$ Department of Statistics Nnamdi Azikiwe University Awka, Anambra State \\ ${ }^{3}$ Department of Statistics, Imo State University, Owerri, PMB 2000, Owerri Nigeria
}

Email address:

fedocon2003@yahoo.com(N. F. Dozie),danekezie@yahoo.com(E. D. Dan)

\section{To cite this article:}

Nwosu F. Dozie, Onyeagu I. Sidney, Osuji A. George, Ekezie Dan Dan. Method of Maximum Likelihood Estimation of Optimal Number of Factors: An Information Criteria Approach. American Journal of Theoretical and Applied Statistics. Vol. 2, No. 6, 2013 , pp. 191-201. doi: 10.11648/j.ajtas.20130206.16

\begin{abstract}
In this paper, we established the number of factors $(\mathrm{k})$ to retain in a factor analysis for different sample sizes for the method of maximum likelihood estimation using StataSE 9 package. Using simulated values, the Akaike (AIC), the Schwarz (SIC) and Hannan Quinne (HQIC) Information criteria values are obtained for samples of 30, 50, and 70 when the number of variables considered is 10 and the number of factors to retain are 2,3, and 5. It was discovered that the AIC, SIC and HQIC values are smallest when $\mathrm{k}$ is 5 and highest when $\mathrm{k}$ is 2 . This implies that the optimal number of factors to retain is 5. Also, as the sample size increases, the AIC, SIC and HQIC for all the k values increases.
\end{abstract}

Keywords: Factor Analysis, Factor Rotation, Maximum Likelihood Estimation Method, Akaike, Schwarz, Hannan Quinne Information Criteria

\section{Introduction}

Factor analysis attempts to simplify complex and diverse relationships that exist among a set of observed variables by uncovering common dimensions or factors that link together the seemingly unrelated variables and consequently provides insight into the underlying structure of the data. The goal of factor analysis is to reduce a large number of variables to a smaller number of factors, to concisely describe the relationship among observed variables or to test theory about underlying processes (Comrey and Lee; 1992).

Factor analysis can be viewed as an extension of principal components analysis. Both are attempts to approximate the covariance matrix, $\Sigma$. principal components analysis and factor-analytic model often yield solutions that are very similar. For this reason, many authors treat principal components analysis as just another many authors type of factor analysis. Under the factor model, each response variates will be represented as a linear function of a small number of unobservable commonfactor variates and a single latent specific variates. The common factors generate the covariances among the observable responses, while the specific terms contribute only to the variances of their particular responses.

\subsection{The Mathematical Model for Factor Structure}

Suppose that the multivariate system consists of $p$ responses described by the observable random variables $\mathrm{X}_{1}, \ldots \mathrm{Xp}$. The $\mathrm{Xi}$ have a non-singular multinormal distribution. Since only the covariance structure will be of interest, we can assume without loss of generality that the population means of $X_{i}$ are zero (Onyegu;2003).

Let,

$$
\begin{gathered}
\mathrm{Y}_{1}-\mu_{1}=\lambda_{11} \mathrm{X}_{1}+\cdots+\lambda_{1 \mathrm{~m}} \mathrm{X}_{m}+e_{1} \\
\vdots \\
\mathrm{Y}_{\mathrm{p}}-\mu_{\mathrm{p}}=\lambda_{\mathrm{p} 1} \mathrm{X}_{1}+\cdots+\lambda_{\mathrm{pm}} \mathrm{X}_{m}+e_{\mathrm{p}}
\end{gathered}
$$

where,

$\mathrm{X}_{\mathrm{j}}=\mathrm{j}$-th common-factor variates

$\lambda_{\mathrm{ij}}=$ parameter reflecting importance of the $\mathrm{j}$-th factor in composition of ith response

$\varepsilon_{1}=$ ith specific factor variates.

In the usage of factor analysts, $\lambda_{\text {ij }}$ is called the loading of the ith response on the jth common factor.

For matrix version of the model;

Let,

$$
X^{\prime}=\left(x_{1}, x_{2}, \ldots, x_{m}\right), \quad y^{\prime}=\left(y_{1}, y_{2}, \ldots, y_{p}\right)
$$


$\varepsilon^{\prime}=\left(\varepsilon_{1}, \varepsilon_{2}, \ldots, \varepsilon_{\mathrm{p}}\right), \quad \mu^{\prime}=\left(\mu_{1}, \mu_{2}, \ldots, \mu_{\mathrm{p}}\right)$,

and

$$
\Lambda=\left[\begin{array}{ccc}
\lambda_{11} & . & \lambda_{1 \mathrm{~m}} \\
: & : & : \\
\lambda_{\mathrm{p} 1} & . . & \lambda_{\mathrm{pm}}
\end{array}\right] .
$$

Then, the factor model can be written as

$$
\left(y_{\mathrm{i}}-\mu_{\mathrm{y}}\right)=\Lambda \mathrm{X}_{\mathrm{i}}+\varepsilon_{\mathrm{i}}, \mathrm{i}=1, \ldots, \mathrm{q} \text {. }
$$

Where $\mathrm{q}$ is the number of observations. $\mathrm{Y}_{\mathrm{i}}$ is an observed vector with $\mathrm{p}$ components with mean $\mu_{y}$ and $\Lambda$ is a $p \times q$ matrix called the factor loadings. $\mathrm{X}_{\mathrm{i}}$ is an observed vector with $q$ components, $q<p$, the components of which are called common factors and $\varepsilon_{\mathrm{i}}$ is an unobserved error with mean $0_{(\mathrm{px} 1)}$ and is called the specific factor. $X_{i}$ is assumed to follow a normal distribution with

$\mathrm{E}\left(\mathrm{X}_{\mathrm{i}}\right)=\mathrm{O}_{(\mathrm{q} \times 1)}, \operatorname{Var}\left(\mathrm{X}_{\mathrm{i}}\right)=\mathrm{E}\left(\mathrm{X}_{\mathrm{i}} \mathrm{X}_{\mathrm{i}}^{\prime}\right)=\mathrm{I}_{(\mathrm{q} \times \mathrm{q})}$, that is the orthogonal case.

Then, $\mathrm{Y}_{\mathrm{i}} / \mathrm{X}_{\mathrm{i}}$ follows $N\left(\mu_{\mathrm{y}}+\Lambda \mathrm{X}_{\mathrm{i}}, \operatorname{Cov}\left(\varepsilon_{\mathrm{i}}\right)\right)$, where $\operatorname{Cov}\left(\varepsilon_{\mathrm{i}}\right)=\psi^{2}=\operatorname{diag}\left(\psi_{1}^{2}, \ldots, \psi_{\mathrm{p}}^{2}\right)$.

$\psi^{2}$ is called uniqueness or specific variance.

Consequently, a covariance structure for $\mathrm{Y}$ is

$\Sigma=\operatorname{Cov}\left(Y_{\mathrm{i}}\right)=\mathrm{E}\left(Y_{\mathrm{i}}-\mu_{\mathrm{y}}\right)\left(Y_{\mathrm{i}}-\mu_{\mathrm{y}}\right)^{\prime}$

$=\mathrm{E}\left(\Lambda \mathrm{X}_{\mathrm{i}}+\varepsilon_{\mathrm{i}}\right)\left(\Lambda \mathrm{X}_{\mathrm{i}}+\varepsilon_{\mathrm{i}}\right)^{\prime}=\mathrm{E}\left(\Lambda \mathrm{X}_{\mathrm{i}} \mathrm{X}_{\mathrm{i}}^{\prime} \Lambda^{\prime}+\right.$

$\left.\varepsilon_{\mathrm{i}} \mathrm{X}_{\mathrm{i}}^{\prime} \Lambda^{\prime}+\Lambda \mathrm{X}_{\mathrm{i}} \varepsilon^{\prime}{ }_{\mathrm{i}}+\varepsilon_{\mathrm{i}} \varepsilon_{\mathrm{i}}^{\prime}\right) \quad=\mathrm{E}\left(\Lambda \mathrm{X}_{\mathrm{i}} \mathrm{X}_{\mathrm{i}}^{\prime} \Lambda^{\prime}\right)+$

$\mathrm{E}\left(\varepsilon_{\mathrm{i}} \mathrm{X}_{\mathrm{i}}^{\prime} \Lambda^{\prime}\right)+\mathrm{E}\left(\Lambda \mathrm{X}_{\mathrm{i}} \varepsilon_{\mathrm{i}}^{\prime}\right)+\mathrm{E}\left(\varepsilon_{\mathrm{i}} \varepsilon_{\mathrm{i}}^{\prime}\right)$ Recall that,

$\mathrm{E}\left(\mathrm{X}_{\mathrm{i}}\right)=0_{(\mathrm{qx} 1)}, \mathrm{E}\left(\mathrm{X}_{\mathrm{i}} \mathrm{X}_{\mathrm{i}}^{\prime}\right)=\mathrm{I}_{(\mathrm{qxq})}, \operatorname{Cov}\left(\varepsilon_{\mathrm{i}}\right)=\mathrm{E}\left(\varepsilon_{\mathrm{i}} \varepsilon_{\mathrm{i}}^{\prime}\right)=$ $\psi^{2}$,

Then, $\mathrm{E}\left(\Lambda \mathrm{X}_{\mathrm{i}} \mathrm{X}_{\mathrm{i}}^{\prime} \Lambda^{\prime}\right)+\mathrm{E}\left(\varepsilon_{\mathrm{i}} \mathrm{X}_{\mathrm{i}}^{\prime} \Lambda^{\prime}\right)+\mathrm{E}\left(\Lambda \mathrm{X}_{\mathrm{i}}{\varepsilon_{\mathrm{i}}^{\prime}}^{\prime}\right)+$ $\mathrm{E}\left(\varepsilon_{\mathrm{i}} \varepsilon_{\mathrm{i}}^{\prime}\right)=\Lambda \Lambda^{\prime}+\psi^{2}$, and

$\operatorname{Cov}\left(Y_{\mathrm{i}}, \mathrm{X}_{\mathrm{i}}\right)=\mathrm{E}\left(Y_{\mathrm{i}}-\mu_{\mathrm{y}}\right) \mathrm{X}_{\mathrm{i}}^{\prime}=\mathrm{E}\left(\Lambda \mathrm{X}_{\mathrm{i}}+\varepsilon_{\mathrm{i}}\right) \mathrm{X}_{\mathrm{i}}^{\prime}$.

Introducing communality, $\operatorname{Cov}\left(\mathrm{Y}_{\mathrm{i}}\right)=\Lambda \Lambda^{\prime}+\psi^{2}$ can be written as

$\operatorname{Var}\left(Y_{i j}\right)=\Lambda_{1 j}^{2}+\ldots+\Lambda_{q j}^{2}+\Psi_{j}^{2} ; \operatorname{Cov}\left(Y_{i j}, Y_{i k}\right)=$ $\Lambda_{1 j} \Lambda_{1 k}+\ldots+\Lambda_{q j} \Lambda_{q k}$ and

$\operatorname{Cov}\left(Y_{i k}, X_{j k}\right)=\Lambda_{j k}$.

Communality is the portion of the variance of the variable contributed by the q common factors.

Suppose the $\mathrm{jth}$ communality is $\mathrm{h}_{j}^{2}$, then

$$
\begin{gathered}
\operatorname{Var}\left(Y_{\mathrm{ij}}\right)=\sigma_{\mathrm{ij}}=\text { communality }+ \text { specific variance }= \\
\mathrm{h}_{\mathrm{j}}^{2}+\Psi_{\mathrm{j}}=\left(\lambda_{1 \mathrm{j}}^{2}+\lambda_{2 \mathrm{j}}^{2}+\cdots+\lambda_{\mathrm{qj}}^{2}\right)+\psi_{\mathrm{j}}, \mathrm{j}=1, \ldots, \mathrm{p} .
\end{gathered}
$$

The $j$-th communality is the sum of square of the loading of the $\mathrm{jth}$ variable on the $\mathrm{q}$ common factor. When the number of factors $\mathrm{q}>1$, there are multiple factor loadings that generate the same covariance matrix.

The loading in the model above can be multiply by an orthogonal matrix without impairing their ability to reproduce the covariance matrix in $\Sigma=\Lambda \Lambda^{\prime}+\psi^{2}$.

Let $\beta$ be any qxq orthogonal matrix. If we let $\Lambda^{*}=\Lambda \beta$ and $\mathrm{X}_{\mathrm{i}}{ }^{*}=\beta^{\prime} \mathrm{X}_{\mathrm{i}}$, then $\mathrm{X}_{\mathrm{i}}{ }^{*}$ has the same statistical properties as $X_{i}$ since

$\mathrm{E}\left(\mathrm{X}_{\mathrm{i}}^{*}\right)=E\left(\beta^{\prime} \mathrm{X}_{\mathrm{i}}\right)=\beta^{\prime} \quad E\left(\mathrm{X}_{\mathrm{i}}\right)=0$.

$\operatorname{Cov}\left(\mathrm{X}_{\mathrm{i}}^{*}\right)=\operatorname{Cov}\left(\beta^{\prime} \mathrm{X}_{\mathrm{i}}\right)=\beta^{\prime} \quad \operatorname{Cov}\left(\mathrm{X}_{\mathrm{i}}\right) \beta=\beta^{\prime} \quad \beta=\mathrm{I}_{\mathrm{mxm}}$

$\Lambda$ and $\Lambda^{\prime}$ yield the same covariance because $\Lambda \Lambda^{\prime}=$ $\Lambda^{*} \Lambda^{* \prime}$. The factor model

$\left(Y_{\mathrm{i}}-\mu_{\mathrm{y}}\right)=\Lambda \mathrm{X}_{\mathrm{i}}+\varepsilon_{\mathrm{i}}=\Lambda \beta \beta^{\prime} \mathrm{X}_{\mathrm{i}}+\varepsilon_{\mathrm{i}}=\Lambda^{*} \mathrm{X}_{\mathrm{i}}^{*}+\varepsilon_{\mathrm{i}}$, produces the same covariance matrix $\Sigma$, since $\Sigma=$ $\Lambda \Lambda^{\prime}+\psi^{2}=\Lambda \beta \beta^{\prime} \quad \Lambda+\psi^{2}=\Lambda^{*} \Lambda^{* \prime}+\psi^{2}$.

A particular set of loadings needs to be chosen. A good set is one that is easily interpreted. This means sparse solution with many zero. Factor rotation is one approval to finding the solution, as it rotates the coordinates system for $\mathrm{Y}$ on $\mathrm{X}$.

In this work, we consider four kinds of orthogonal factor rotations namely, varimas, equmax, quartimax and orthomax.

\section{The Maximum Likelihood Method}

If the distribution of $X$ and the specific factors, $\varepsilon_{\mathrm{i}}$, are assumed to be normal, the estimates of the factor loadings and the uniqueness can be obtained using the maximum likelihood method (Johnson and Winchern, 2007). The distribution of $\mathrm{Y}$ given $\Lambda, \psi^{2}$ is normal with mean 0 and covariance matrix $\Lambda \Lambda^{\prime}+\psi^{2}$, and the likelihood is

$$
L\left(\Lambda, \Psi^{2}\right) \propto \operatorname{det}(\Sigma)^{-\frac{\mathrm{n}}{2}} \mathrm{e}^{-\frac{1}{2}} \sum_{i=1}^{n}\left(y_{i}-\mu_{\mathrm{y}}\right) \Sigma^{-1}\left(y_{i}-\mu_{\mathrm{y}}\right)^{\prime} .
$$

The resulting log likelihood (LL) is

$$
L L(\Lambda, \Psi) \propto-\frac{\mathrm{n}}{2} \operatorname{In} \operatorname{det}(\Sigma)-\frac{1}{2} \sum_{i=1}^{n}\left(y_{i}-\mu_{\mathrm{y}}\right) \Sigma^{-1}\left(y_{i}-\right.
$$
$\left.\mu_{\mathrm{y}}\right)^{\prime}$

The maximum likelihood estimates of $\Lambda, \psi^{2}$ (called $\widehat{\Lambda}$ and $\widehat{\psi}^{2}$ ) can be obtained by maximizing the LL. It can be shown that the estimates $\widehat{\Lambda}$ and $\widehat{\psi}^{2}$ satisfy the following;

$$
S \widehat{\psi}^{2} \widehat{\Lambda}=\widehat{\Lambda}\left(\mathrm{I}+\widehat{\Lambda}^{\prime} \quad\left(\widehat{\Psi}^{2}\right)^{-1} \widehat{\Lambda}\right) .
$$

$\widehat{\psi}^{2}=\operatorname{diag}\left(S-\widehat{\Lambda} \widehat{\Lambda}^{\prime}\right), \widehat{\Lambda}^{\prime}\left(\widehat{\psi}^{2}\right)^{-1} \widehat{\Lambda}$ is diagonal.

Based on the invariance property of maximum likelihood estimates, the maximum likelihood estimate of the communality due to $j$-th factor is $\hat{\lambda}_{1 j}^{2}+\hat{\lambda}_{2 j}^{2}+\cdots+\hat{\lambda}_{\mathrm{qj}}^{2}$.

As a result, the proportion of the total sample variance due the $\mathrm{j}$-th factor is $\frac{\hat{\lambda}_{\mathrm{j}}^{2}+\hat{\lambda}_{2 \mathrm{j}}^{2}+\cdots+\hat{\lambda}_{\mathrm{qj}}^{2}}{S_{11}+S_{22}+\cdots+S_{q q}}$, where $S_{i i}$ is the (i,i)-th entry of the sample covariance matrix which is an estimated of the unknown population covariance matrix, $\Sigma$ 
If $y_{i}$ is standardized to be

$$
Z_{i}=\mathrm{V}^{-\frac{1}{2}}\left(y_{i}-\mu_{y}\right)
$$

the covariance matrix $\mathrm{q}$ is given as

$Q=\mathrm{V}^{-\frac{1}{2}} \Sigma \mathrm{V}^{-\frac{1}{2}}=\left(\mathrm{V}^{-\frac{1}{2}} \Lambda\right)\left(\mathrm{V}^{-\frac{1}{2}} \Lambda\right)^{\prime}+\mathrm{V}^{-\frac{1}{2}} \widehat{\Psi}^{2} \mathrm{~V}^{-\frac{1}{2}}=$ $\Lambda_{z} \Lambda_{z}^{\prime}+\widehat{\psi}^{2}{ }^{\prime}$, where $\mathrm{V}^{-\frac{1}{2}}$ is the diagonal matrix with the reciprocal of the sample standard deviation on the main diagonal of $y_{i}$. Based on the invariance property of maximum likelihood estimators, the maximum likelihood estimator of $Q$ is

$$
Q=\widehat{\Lambda}_{z} \widehat{\Lambda}_{z}^{\prime}+\widehat{\Psi}^{2}{ }^{\prime} \text {, where } \widehat{\Lambda}_{z}=\mathrm{V}^{-\frac{1}{2}} \widehat{\Lambda} \text {. }
$$

The proportion of total standardized sample variance due to $j$-th factor is

$\frac{\sum_{i=1}^{q} \widehat{\lambda}_{\mathrm{ij}}^{2}}{\operatorname{tr}(S)}=\frac{\widehat{\lambda}_{1 \mathrm{j}}^{2}+\hat{\lambda}_{2 \mathrm{j}}^{2}+\cdots+\hat{\lambda}_{\mathrm{qj}}^{2}}{S_{11}+S_{22}+\cdots+S_{q q}}=\frac{\widehat{\lambda}_{1 \mathrm{j}}^{2}+\hat{\lambda}_{2 \mathrm{j}}^{2}+\cdots+\hat{\lambda}_{\mathrm{qj}}^{2}}{q}$, where $S_{i i}=\hat{\lambda}_{1 \mathrm{j}}^{2}+$
$\widehat{\Psi}_{1 \mathrm{j}}^{2}$.

\subsection{Information Criteria}

The necessity of introducing the concept of model evaluation has been recognized as one of the important technical areas and the problem is posed on the choice of the best approximating model among a class of competing models by a suitable model evaluation criterion given a data set. Model evaluation criteria are figures of merit, or performance measures for competing models. Factor analysis can be characterized as multivariate technique for analyzing the internal relationship among a set of variables. Based on the usual factor analysis model, choosing a model with too few parameters can involve making unrealistically simple assumptions and lead to high bias, poor prediction, and missed opportunities for insight. Such models are not flexible enough to describe the sample or the population well. A model with too many parameters can fit the observed data very well, but be too closely tailored to it; such models may generalize poorly. Penalized-likelihood information criteria, such as Akaike's information criterion (AIC), the Schwarz's information criterion (SIC), the
Hannan-Quinn information criterion (HQIC) and so on are widely used for model selection. The comparison of the models using information criterion can be viewed as equivalent to a likelihood ratio test and understanding the differences among the criteria may make it easier to compare the results and to use them to make informed decisions (Akaike; 1973).

AKAIKE'S information criterion is probably the most relevant and famous as for the comparison and selection between different models and is constructed on log likelihood

$$
\mathrm{AIC}=-2 \log \max L+2 k
$$

where $L$ denotes the likelihood function of the factor model and $k$ is the number of the model's parameter/factors. $\log \max L(k)=-\frac{1}{2} N|\log | \Sigma_{k}\left|+\operatorname{tr} \Sigma_{k}^{-1} S\right|$, where $S$ denotes the sample covariance matrix of $\mathrm{Y}$ and $\Sigma_{k}=$ $\Lambda_{k} \Lambda_{k}^{-1}+\psi^{2} ; \Lambda_{k}$ is the matrix factor of factor loading. The first term can be interpreted as a goodness-of-fit measurement, while the second gives a growing penalty to increasing numbers of parameters according to the parsimony principle. In the choice of model, a minimization rule is used to select the model with the minimum Akaike information criterion value.

Still in the likelihood based procedures, Schwarz (1978) proposed the alternative information criterion given by

$$
\text { SIC }=-\log \max L+\frac{1}{2} k \log N .
$$

Unlike the AIC. SIC considers the number of $N$ of observations and is therefore less favourable to factors inclusion.

Finally, the third criteria are the Hannan-Quinn information criterion (HQC); it is a criterion for model selection. It is an alternative to Akaike information criterion (AIC) and Bayesian information criterion (BIC)(Harman;1976). It is given as

$$
H Q I C=-\log \max L+2 k \log \log N
$$

where $k$ is the number of parameters, $N$ is the number of observations..

\section{Comparison of AIC and SIC after Rotation at Different Sample Sizes and Different Retained Number of Factors $(k)$}

3.1. For $n=30, p=10$ and $k=2$

\subsubsection{Rotated Factor Loadings}

\begin{tabular}{ccccccccc}
\hline \multicolumn{2}{c}{ Varimax Loadings } & \multicolumn{2}{c}{ Equamax Loadings } & \multicolumn{2}{c}{ Quartimax Loadings } & \multicolumn{2}{c}{ Orthomax Loadings } \\
$\mathbf{I}$ & II & I & II & I & II & I & II \\
\hline-0.3702 & -0.2308 & -0.3577 & -0.2347 & -0.3609 & -0.2297 & -0.3552 & -0.2385 \\
-0.4130 & 0.2369 & -0.4155 & 0.2311 & -0.4122 & 0.2369 & -0.4179 & 0.2267 \\
0.1917 & 0.9809 & 0.1811 & 0.9835 & 0.1948 & 0.9809 & 0.1707 & 0.9853 \\
0.9773 & 0.2086 & 0.9750 & 0.2222 & 0.9780 & 0.2086 & 0.9726 & 0.2325 \\
0.3106 & -0.2791 & 0.3136 & -0.2747 & 0.3098 & -0.2786 & 0.3165 & -0.2714 \\
\hline
\end{tabular}




\begin{tabular}{ccccccccc}
\hline \multicolumn{2}{c}{ Varimax Loadings } & \multicolumn{2}{c}{ Equamax Loadings } & \multicolumn{2}{c}{ Quartimax Loadings } & \multicolumn{2}{c}{ Orthomax Loadings } \\
$\mathbf{I}$ & II & I & II & I & I & I & I \\
\hline 0.2343 & -0.1618 & 0.2361 & -0.1586 & 0.2338 & -0.1618 & 0.2377 & -0.1561 \\
0.0613 & 0.1670 & 0.0595 & 0.1678 & 0.0618 & 0.1670 & 0.0577 & 0.1684 \\
-0.1235 & -0.1640 & -0.1217 & -0.1657 & -0.1240 & -0.1640 & -0.1200 & -0.1670 \\
0.1354 & 0.2694 & 0.1324 & 0.2713 & 0.1362 & 0.2694 & 0.1296 & 0.2727 \\
0.1303 & -0.1413 & 0.1318 & -0.1395 & 0.1299 & -0.1413 & 0.1333 & -0.1381 \\
\hline
\end{tabular}

\subsubsection{Factor Rotation Matrix}

Varimax

\begin{tabular}{lll}
\hline & Factor I & Factor II \\
\hline Factor I & 0.4587 & 0.8886 \\
Factor II & 0.8886 & -0.4587 \\
\hline
\end{tabular}

Equamax

\begin{tabular}{lll}
\hline & Factor I & Factor II \\
\hline Factor I & 0.4490 & 0.8935 \\
Factor II & 0.8935 & -0.4490 \\
\hline
\end{tabular}

Quartimax

\begin{tabular}{lll}
\hline & Factor I & Factor II \\
\hline Factor I & 0.4614 & 0.8872 \\
Factor II & 0.8872 & -0.4614 \\
\hline
\end{tabular}

\subsection{For $n=30, p=10$ and $k=3$}

Orthomax

\begin{tabular}{lll}
\hline & Factor I & Factor II \\
\hline Factor I & 0.4396 & 0.8982 \\
Factor II & 0.8982 & -0.4396 \\
\hline
\end{tabular}

\subsubsection{Information Criteria}

\begin{tabular}{ll}
\hline Information Criteria & Values \\
\hline Log Likelihood & -116.6265 \\
Akaike & 237.2530 \\
Schwarz & 118.1036 \\
Hannan Quinne & 117.3042
\end{tabular}

\subsubsection{Rotated Factor Loadings}

\begin{tabular}{|c|c|c|c|c|c|c|c|c|c|c|c|}
\hline \multicolumn{3}{|c|}{ Varimax Loadings } & \multicolumn{3}{|c|}{ Equamax Loadings } & \multicolumn{3}{|c|}{ Quartimax Loadings } & \multicolumn{3}{|c|}{ Orthomax Loadings } \\
\hline $\begin{array}{c}\text { Factor } \\
\text { I } \\
\end{array}$ & $\begin{array}{c}\text { Factor } \\
\text { II } \\
\end{array}$ & $\begin{array}{c}\text { Factor } \\
\text { III } \\
\end{array}$ & $\begin{array}{c}\text { Factor } \\
\text { I } \\
\end{array}$ & $\begin{array}{c}\text { Factor } \\
\text { II } \\
\end{array}$ & $\begin{array}{c}\text { Factor } \\
\text { III } \\
\end{array}$ & $\begin{array}{c}\text { Factor } \\
\text { I } \\
\end{array}$ & $\begin{array}{c}\text { Factor } \\
\text { II } \\
\end{array}$ & $\begin{array}{c}\text { Factor } \\
\text { III } \\
\end{array}$ & $\begin{array}{c}\text { Factor } \\
\text { I } \\
\end{array}$ & $\begin{array}{c}\text { Factor } \\
\text { II } \\
\end{array}$ & $\begin{array}{c}\text { Factor } \\
\text { III } \\
\end{array}$ \\
\hline-0.3596 & -0.2388 & 0.1169 & -0.3566 & -0.2458 & 0.1116 & -0.3605 & -0.2369 & 0.1181 & -0.3534 & -0.2497 & 0.1128 \\
\hline-0.4125 & 0.2337 & 0.0431 & -0.4154 & 0.2275 & 0.0481 & -0.4117 & 0.2354 & 0.0418 & -0.4186 & 0.2222 & 0.0456 \\
\hline 0.1929 & 0.9791 & 0.0647 & 0.1805 & 0.9798 & 0.0859 & 0.1962 & 0.9788 & 0.0596 & 0.1671 & 0.9828 & 0.0788 \\
\hline 0.9776 & 0.2104 & 0.0079 & 0.9748 & 0.2226 & 0.0127 & 0.9783 & 0.2071 & 0.0070 & 0.9717 & 0.2358 & 0.0129 \\
\hline 0.3121 & -0.2970 & 0.2662 & 0.3159 & -0.2987 & 0.2598 & 0.3111 & -0.2967 & 0.2678 & 0.3193 & -0.2925 & 0.2626 \\
\hline 0.2339 & -0.1580 & -0.0552 & 0.2358 & -0.1538 & -0.0586 & 0.2333 & -0.1591 & -0.0544 & 0.2380 & -0.1511 & -0.0570 \\
\hline 0.0656 & 0.1231 & 0.6598 & 0.0641 & 0.1096 & 0.6623 & 0.0660 & 0.1263 & 0.6592 & 0.0613 & 0.1154 & 0.6616 \\
\hline-0.1223 & -0.1792 & 0.2167 & -0.1200 & -0.1854 & 0.2127 & -0.1229 & -0.1777 & 0.2176 & -0.1179 & -0.1854 & 0.2139 \\
\hline 0.1375 & 0.2498 & 0.3046 & 0.1344 & 0.2449 & 0.3100 & 0.1383 & 0.2509 & 0.3033 & 0.1304 & 0.2490 & 0.3084 \\
\hline 0.1267 & -0.1041 & -0.5539 & 0.1280 & -0.0905 & -0.5560 & 0.1264 & -0.1074 & -0.5533 & 0.1303 & -0.0930 & -0.5550 \\
\hline
\end{tabular}

\subsubsection{Factor Rotation Matrix}

Varimax

\begin{tabular}{|c|c|c|c|}
\hline & Factor I & Factor II & Factor III \\
\hline Factor I & 0.9598 & 0.2805 & 0.0128 \\
\hline Factor II & -0.2807 & 0.9575 & 0.0662 \\
\hline Factor III & 0.0063 & -0.0671 & 0.9977 \\
\hline \multicolumn{4}{|c|}{ Equamax } \\
\hline & Factor I & Factor II & Factor III \\
\hline Factor I & 0.9562 & 0.2922 & 0.0191 \\
\hline Factor II & -0.2928 & 0.9522 & 0.0868 \\
\hline Factor III & 0.0072 & -0.0886 & 0.9960 \\
\hline
\end{tabular}

Quartimax

\begin{tabular}{cccc}
\hline & Factor I & Factor II & Factor III \\
\hline Factor I & 0.9607 & 0.2772 & 0.0115 \\
Factor II & -0.2774 & 0.9588 & 0.0612 \\
Factor III & 0.0059 & -0.0620 & 0.9981 \\
\hline \multicolumn{4}{c}{ Orthomax } \\
\hline Factor I & Factor II & Factor III \\
\hline Factor I & 0.9521 & 0.3052 & 0.0187 \\
Factor II & -0.3057 & 0.9488 & 0.0791 \\
Factor III & 0.6452 & -0.0810 & 0.9967 \\
\hline
\end{tabular}


3.2.3. Information Criteria

\begin{tabular}{cc}
\hline Information Criteria & Values \\
\hline Log Likelihood & -112.6245 \\
Akaike & 231.2490 \\
Schwarz & 114.8402 \\
Hannan Quinne & 109.9072 \\
\hline
\end{tabular}

\subsection{For $n=30, p=10$, and $k=5$}

\subsubsection{Rotated Factor Loadings}

\begin{tabular}{|c|c|c|c|c|c|c|c|c|c|}
\hline \multicolumn{5}{|c|}{ Varimax Loadings } & \multicolumn{5}{|c|}{ Equamax Loadings } \\
\hline 1 & 2 & 3 & 4 & 5 & 1 & 2 & 3 & 4 & 5 \\
\hline-0.3048 & -0.1789 & -0.1510 & -0.3019 & 0.3626 & -0.2696 & -0.2996 & -0.1599 & -0.1268 & 0.4077 \\
\hline 0.2245 & 0.0814 & 0.0675 & -0.6254 & 0.0872 & 0.2542 & -0.6117 & 0.0910 & 0.0727 & 0.0889 \\
\hline 0.9738 & -0.1255 & -0.0744 & 0.0788 & -0.0250 & 0.9665 & 0.1128 & -0.1273 & -0.0839 & -0.0799 \\
\hline 0.3850 & 0.2427 & 0.1088 & 0.7501 & 0.0204 & 0.3552 & 0.7662 & 0.2363 & 0.1027 & -0.0492 \\
\hline-0.1048 & 0.9879 & -0.0852 & 0.0734 & -0.0219 & -0.1122 & 0.0757 & 0.9852 & -0.0880 & -0.0570 \\
\hline-0.1445 & -0.1293 & 0.1336 & 0.3524 & 0.2666 & -0.1405 & 0.3567 & -0.1207 & 0.1479 & 0.2594 \\
\hline 0.1798 & 0.1513 & -0.3317 & 0.0477 & 0.3284 & 0.1985 & 0.0681 & 0.1652 & -0.3154 & 0.3250 \\
\hline-0.2144 & -0.2273 & -0.1283 & -0.0367 & 0.4503 & -0.1843 & -0.0283 & -0.2068 & -0.1011 & 0.4801 \\
\hline 0.3184 & 0.1505 & -0.0711 & 0.0362 & 0.3408 & 0.3364 & 0.0629 & 0.1651 & -0.0557 & 0.3147 \\
\hline-0.0542 & -0.0761 & 0.9944 & 0.0391 & -0.0304 & -0.0524 & 0.0376 & -0.0778 & 0.9917 & -0.0796 \\
\hline \multicolumn{5}{|c|}{ Quartimax Loadings } & \multicolumn{5}{|c|}{ Orthomax Loadings } \\
\hline 1 & 2 & 3 & 4 & 5 & 1 & 2 & 3 & 4 & 5 \\
\hline-0.3124 & -0.1831 & -0.1571 & -0.2980 & 0.3545 & -0.2443 & -0.1536 & -0.1189 & -0.1712 & 0.4935 \\
\hline 0.2156 & 0.0778 & 0.0654 & -0.6289 & 0.0893 & 0.2799 & 0.0927 & 0.0769 & -0.5509 & 0.2527 \\
\hline 0.9749 & -0.1263 & -0.0731 & 0.0670 & -0.0170 & 0.9550 & -0.1374 & -0.1013 & 0.0990 & -0.1598 \\
\hline 0.3945 & 0.2448 & 0.1101 & 0.7439 & 0.0306 & 0.3308 & 0.2290 & 0.0843 & 0.7248 & -0.2960 \\
\hline-0.1024 & 0.9885 & -0.0848 & 0.0711 & -0.0145 & -0.1102 & 0.9851 & -0.0904 & 0.0549 & -0.0789 \\
\hline-0.1430 & -0.1304 & 0.1301 & 0.3537 & 0.2669 & -0.1388 & -0.1192 & 0.1441 & 0.4174 & 0.1492 \\
\hline 0.1775 & 0.1481 & -0.3362 & 0.0444 & 0.3269 & 0.2039 & 0.1641 & -0.3217 & 0.1611 & 0.2781 \\
\hline-0.2197 & -0.2314 & -0.1351 & -0.0342 & 0.4438 & -0.1648 & -0.2022 & -0.0989 & 0.1111 & 0.4775 \\
\hline 0.3155 & 0.1470 & -0.0758 & 0.0307 & 0.3445 & 0.3485 & 0.1635 & -0.0644 & 0.1597 & 0.2621 \\
\hline-0.0544 & -0.0758 & 0.9947 & 0.0385 & -0.0170 & -0.0411 & -0.0748 & 0.9924 & 0.1269 & -0.0848 \\
\hline
\end{tabular}

\subsubsection{Factor Rotation Matrix}

Varimax

\begin{tabular}{lccccc}
\hline & Factor 1 & Factor 2 & Factor 3 & Factor 4 & Factor 5 \\
\hline Factor 1 & -0.0891 & 0.9550 & -0.2756 & 0.0622 & -0.0149 \\
Factor 2 & -0.0897 & 0.2632 & 0.9577 & 0.0640 & -0.0377 \\
Factor 3 & 0.9825 & 0.0973 & 0.0541 & 0.1444 & -0.0376 \\
Factor 4 & -0.1320 & -0.0911 & -0.0521 & 0.9853 & 0.0263 \\
Factor 5 & 0.0357 & 0.0302 & 0.0355 & -0.0171 & 0.9981 \\
\hline
\end{tabular}

Equamax

\begin{tabular}{lccccc}
\hline & Factor 1 & Factor 2 & Factor 3 & Factor 4 & Factor 5 \\
\hline Factor 1 & -0.0966 & 0.0647 & 0.9528 & -0.2778 & -0.0387 \\
Factor 2 & -0.0905 & 0.0633 & 0.2607 & 0.9540 & -0.0986 \\
Factor 3 & 0.9717 & 0.1802 & 0.0942 & 0.0428 & -0.1124 \\
Factor 4 & -0.1691 & 0.9791 & -0.0990 & -0.0535 & 0.0041 \\
Factor 5 & 0.0984 & 0.0253 & 0.0744 & 0.0894 & 0.9880 \\
\hline
\end{tabular}

Quartimax

\begin{tabular}{lllclll}
\hline & Factor $\mathbf{1}$ & Factor 2 & Factor 3 & \multicolumn{2}{c}{ Factor 4 } & factor 5 \\
\hline Factor 1 & -0.0868 & 0.9555 & -0.2753 & 0.0601 & -0.0105 \\
Factor 2 & -0.0892 & 0.2638 & 0.9582 & 0.0626 & -0.0219 \\
Factor 3 & 0.9847 & 0.0969 & 0.0558 & 0.1315 & -0.0252 \\
Factor 4 & -0.1197 & -0.0874 & -0.0510 & 0.9873 & 0.0263 \\
Factor 5 & 0.0251 & 0.0206 & 0.0208 & -0.0206 & 0.9990 \\
\hline
\end{tabular}

Orthomax

\begin{tabular}{lllllll}
\hline & Factor $\mathbf{1}$ & Factor 2 & Factor $\mathbf{3}$ & \multicolumn{2}{c}{ Factor 4 } & Factor 5 \\
\hline Factor 1 & -0.0968 & 0.9521 & -0.2801 & 0.0470 & -0.0585 \\
Factor 2 & -0.0786 & 0.2636 & 0.9539 & 0.0455 & -0.1112 \\
Factor 3 & 0.9605 & 0.0838 & 0.0237 & 0.1564 & -0.2130 \\
Factor 4 & -0.2026 & -0.1028 & -0.0651 & 0.9310 & -0.2780 \\
Factor 5 & 0.1442 & 0.0801 & 0.0826 & 0.3232 & 0.9282 \\
\hline
\end{tabular}


3.3.3. Information Criteria

\begin{tabular}{cc}
\hline Information Criteria & Values \\
\hline Log Likelihood & -108.2130 \\
Akaike & 226.4260 \\
Schwarz & 111.9058 \\
Hannan Quinne & 109.9072 \\
\hline
\end{tabular}

3.4. For $n=50, p=10$, and $k=2$.

3.4.1. Rotated Factor Loadings

\begin{tabular}{|c|c|c|c|c|c|c|c|}
\hline \multicolumn{2}{|c|}{ Varimax Loadings } & \multicolumn{2}{|c|}{ Equamax Loadings } & \multicolumn{2}{|c|}{ Quartimax Loadings } & \multicolumn{2}{|c|}{ Orthomax Loadings } \\
\hline I & II & I & II & I & II & $\mathbf{I}$ & II \\
\hline 0.9922 & -0.1243 & 0.9881 & -0.1540 & 0.9933 & -0.1159 & 0.9934 & -0.1149 \\
\hline-0.2951 & 0.7780 & -0.2717 & 0.7865 & -0.3017 & 0.7755 & -0.3025 & 0.7752 \\
\hline 0.1655 & 0.1709 & 0.1705 & 0.1659 & 0.1640 & 0.1723 & 0.1638 & 0.1725 \\
\hline 0.0827 & 0.0269 & 0.0835 & 0.0244 & 0.0825 & 0.0276 & 0.0825 & 0.0276 \\
\hline-0.0489 & -0.0194 & -0.0494 & -0.0179 & -0.0487 & -0.0198 & -0.0487 & -0.0199 \\
\hline 0.0562 & -0.1489 & 0.0517 & -0.1505 & 0.0575 & -0.1484 & 0.0576 & -0.1483 \\
\hline 0.2249 & -0.2948 & 0.2160 & -0.3014 & 0.2274 & -0.2929 & 0.2277 & -0.2926 \\
\hline-0.2756 & -0.3872 & -0.2871 & -0.3787 & -0.2723 & -0.3895 & -0.2719 & -0.3898 \\
\hline 0.0914 & 0.2652 & 0.0993 & 0.2630 & 0.0891 & 0.2666 & 0.0888 & 0.2667 \\
\hline 0.1112 & -0.0118 & 0.1108 & -0.0152 & 0.1113 & -0.0109 & 0.1113 & -0.0108 \\
\hline
\end{tabular}

\subsubsection{Factor Rotation Matrix}

\begin{tabular}{|c|c|c|}
\hline \multicolumn{3}{|c|}{ Varimax } \\
\hline & Factor I & Factor II \\
\hline Factor I & 0.9922 & -0.1243 \\
\hline Factor II & 0.1243 & 0.9922 \\
\hline \multicolumn{3}{|c|}{ Equamax } \\
\hline & Factor I & Factor II \\
\hline Factor I & 0.9881 & -0.1540 \\
\hline Factor II & 0.1540 & 0.9881 \\
\hline
\end{tabular}

Quartimax

\begin{tabular}{lll}
\hline & Factor I & Factor II \\
\hline Factor I & 0.9933 & -0.1159 \\
Factor II & 0.1159 & 0.9933 \\
\hline
\end{tabular}

\subsection{For $n=50, p=10$, and $k=3$.}

\subsubsection{Rotated Factor Loadings}

\begin{tabular}{|c|c|c|c|c|c|c|c|c|c|c|c|}
\hline \multicolumn{3}{|c|}{ Varimax Loadings } & \multicolumn{3}{|c|}{ Equamax Loadings } & \multicolumn{3}{|c|}{ Quartimax Loadings } & \multicolumn{3}{|c|}{ Orthomax Loadings } \\
\hline $\begin{array}{c}\text { Factor } \\
\text { I }\end{array}$ & $\begin{array}{c}\text { Factor } \\
\text { II }\end{array}$ & $\begin{array}{c}\text { Factor } \\
\text { III }\end{array}$ & $\begin{array}{c}\text { Factor } \\
\text { I }\end{array}$ & Factor II & $\begin{array}{c}\text { Factor } \\
\text { III }\end{array}$ & $\begin{array}{c}\text { Factor } \\
\text { I }\end{array}$ & Factor II & $\begin{array}{c}\text { Factor } \\
\text { III }\end{array}$ & $\begin{array}{c}\text { Factor } \\
\text { I }\end{array}$ & Factor II & $\begin{array}{c}\text { Factor } \\
\text { III }\end{array}$ \\
\hline 0.9998 & -0.0199 & -0.0026 & 0.9998 & -0.0200 & 0.0047 & 0.9998 & -0.0198 & -0.0044 & 0.9996 & -0.0202 & 0.0176 \\
\hline-0.0792 & 0.1098 & -0.1916 & -0.0778 & 0.1101 & -0.1920 & -0.0796 & 0.1097 & -0.1915 & -0.0753 & 0.1087 & -0.1937 \\
\hline 0.0384 & 0.0163 & 0.1952 & 0.0369 & 0.0160 & 0.1955 & 0.0387 & 0.0164 & 0.1951 & 0.0344 & 0.0164 & 0.1959 \\
\hline-0.0707 & -0.2210 & 0.0189 & -0.0709 & -0.2210 & 0.0180 & -0.0707 & -0.2210 & 0.0191 & -0.0711 & -0.2209 & 0.0187 \\
\hline-0.2750 & 0.0242 & -0.2080 & -0.2735 & 0.0246 & -0.2100 & -0.2754 & 0.0241 & -0.2076 & -0.2708 & 0.0232 & -0.2137 \\
\hline-0.1164 & -0.0678 & -0.0494 & -0.1161 & -0.0677 & -0.0504 & -0.1165 & -0.0679 & -0.0492 & -0.7754 & -0.0681 & -0.0514 \\
\hline-0.0205 & 0.9995 & 0.0251 & -0.0205 & 0.9994 & 0.0266 & -0.0205 & 0.9995 & 0.0248 & -0.0206 & 0.9996 & 0.0193 \\
\hline 0.1947 & 0.0172 & 0.1319 & 0.1937 & 0.0169 & 0.1334 & 0.1949 & 0.0172 & 0.1316 & 0.1920 & 0.0178 & 0.1357 \\
\hline 0.1810 & 0.1431 & -0.1012 & 0.11818 & 0.1433 & -0.0997 & 0.1808 & 0.1431 & -0.1016 & 0.1830 & 0.1425 & -0.0983 \\
\hline-0.0067 & 0.0419 & 0.8396 & -0.0129 & 0.0405 & 0.1896 & -0.0052 & 0.0422 & 0.8396 & -0.0236 & 0.0465 & 0.8391 \\
\hline
\end{tabular}


3.5.2. Factor Rotation Matrix

\begin{tabular}{|c|c|c|c|}
\hline \multicolumn{4}{|c|}{ Varimax } \\
\hline & Factor I & Factor II & Factor III \\
\hline Factor I & -0.1719 & 0.9848 & 0.0251 \\
\hline Factor II & 0.9851 & 0.1719 & 0.0022 \\
\hline Factor III & 0.0021 & -0.0251 & 0.9997 \\
\hline \multicolumn{4}{|c|}{ Equamax } \\
\hline & Factor I & Factor II & Factor III \\
\hline Factor I & -0.1720 & 0.9848 & 0.0254 \\
\hline Factor II & 0.9851 & 0.1718 & 0.0097 \\
\hline Factor III & -0.0052 & -0.0267 & 0.9996 \\
\hline
\end{tabular}

3.6. For $n=30, p=10$, and $k=5$.
Quartimax

\begin{tabular}{|c|c|c|c|}
\hline & Factor I & Factor II & Factor III \\
\hline Factor I & -0.1719 & 0.9848 & 0.0250 \\
\hline Factor II & 0.9851 & 0.1720 & 0.0004 \\
\hline Factor III & 0.0039 & -0.0247 & 0.9997 \\
\hline \multicolumn{4}{|c|}{ Orthomax } \\
\hline & Factor I & Factor II & Factor III \\
\hline Factor I & -0.1721 & 0.9850 & 0.0162 \\
\hline Factor II & 0.9849 & 0.1717 & 0.0211 \\
\hline Factor III & -0.0180 & -0.0196 & 0.9996 \\
\hline
\end{tabular}

\begin{tabular}{cc} 
3.5.3. Information Criteria & \\
\hline Information Criteria & Values \\
\hline Log Likelihood & -234.1150 \\
Akaike & 474.2300 \\
Schwarz & 236.6635 \\
Hannan Quinne & 235.4961 \\
\hline
\end{tabular}

\subsubsection{Rotated Factor Loadings}

\begin{tabular}{|c|c|c|c|c|c|c|c|c|c|}
\hline \multicolumn{5}{|c|}{ Varimax Loadings } & \multicolumn{5}{|c|}{ Equamax Loadings } \\
\hline 1 & 2 & 3 & 4 & 5 & 1 & 2 & 3 & 4 & 5 \\
\hline-0.2795 & 0.0571 & -0.0315 & 0.8106 & 0.0238 & -0.2534 & 0.0564 & -0.0282 & 0.8188 & 0.0379 \\
\hline 0.9894 & -0.0043 & -0.0118 & -0.1384 & -0.0412 & 0.9817 & -0.0036 & -0.0120 & -0.1680 & -0.0893 \\
\hline 0.1089 & 0.0651 & -0.1572 & 0.1962 & -0.0836 & 0.1107 & 0.0641 & -0.1569 & 0.1937 & -0.0880 \\
\hline-0.0070 & 0.9996 & -0.0084 & 0.0240 & 0.0014 & -0.0078 & 0.9996 & -0.0083 & 0.0250 & -0.0090 \\
\hline-0.0104 & -0.0081 & 0.9997 & -0.0210 & 0.0008 & -0.0122 & -0.0082 & 0.9996 & -0.0248 & -0.0066 \\
\hline-0.0680 & 0.0455 & 0.0348 & 0.0569 & 0.6576 & -0.0343 & 0.0526 & 0.0403 & 0.0571 & 0.6594 \\
\hline-0.2466 & -0.1801 & -0.1940 & 0.2114 & 0.2730 & -0.2260 & -0.1775 & -0.1911 & 0.2187 & 0.2884 \\
\hline-0.2962 & 0.1142 & 0.0306 & -0.3878 & -0.0122 & -0.3082 & 0.1140 & 0.0287 & -0.3786 & 0.0003 \\
\hline 0.1796 & 0.1563 & 0.1081 & 0.1366 & -0.3720 & 0.1649 & 0.1523 & 0.1058 & 0.1317 & -0.3826 \\
\hline-0.0854 & -0.1362 & -0.0856 & 0.1554 & -0.1272 & -0.0864 & -0.1378 & -0.0860 & 0.1585 & -0.1205 \\
\hline \multicolumn{5}{|c|}{ Quartimax Loadings } & \multicolumn{5}{|c|}{ Orthomax Loadings } \\
\hline 1 & 2 & 3 & 4 & 5 & 1 & 2 & 3 & 4 & 5 \\
\hline-0.2872 & 0.0573 & -0.0325 & 0.8079 & 0.0210 & -0.2816 & 0.0631 & -0.0282 & 0.8083 & 0.0505 \\
\hline 0.9911 & -0.0047 & -0.0120 & -0.1292 & -0.0295 & 0.9815 & -0.0059 & -0.0130 & -0.1312 & -0.1384 \\
\hline 0.1081 & 0.0653 & -0.1573 & 0.1970 & -0.0823 & 0.0989 & 0.0642 & -0.1581 & 0.1970 & -0.0925 \\
\hline-0.0068 & 0.9997 & -0.0085 & 0.0237 & 0.0039 & -0.0102 & 0.9994 & -0.0085 & 0.0172 & -0.0254 \\
\hline-0.0098 & -0.0080 & 0.9997 & -0.0197 & 0.0025 & -0.0122 & -0.0085 & 0.9994 & -0.0246 & -0.0182 \\
\hline-0.0763 & 0.0438 & 0.0335 & 0.0559 & 0.6570 & -0.0033 & 0.0641 & 0.0484 & 0.0562 & 0.6588 \\
\hline-0.2519 & -0.1807 & -0.1947 & 0.2087 & 0.2693 & -0.2189 & -0.1711 & -0.1876 & 0.2116 & 0.3048 \\
\hline-0.2923 & 0.1143 & 0.0313 & -0.3905 & -0.0156 & -0.1936 & 0.1110 & 0.0290 & -0.3907 & 0.0133 \\
\hline 0.1828 & 0.1572 & 0.1085 & 0.1386 & -0.3691 & 0.1402 & 0.1469 & 0.1010 & 0.1363 & -0.3942 \\
\hline-0.0854 & -0.1358 & -0.0855 & 0.1546 & -0.1286 & -0.0980 & -0.1386 & -0.0876 & 0.1560 & -0.1125 \\
\hline
\end{tabular}

\subsubsection{Factor Rotation Matrix}

\section{Varimax}

\begin{tabular}{lccccc}
\hline & Factor 1 & Factor 2 & Factor 3 & Factor 4 & Factor 5 \\
\hline Factor 1 & 0.9894 & -0.0058 & -0.0127 & -0.1384 & -0.0412 \\
Factor 2 & 0.0087 & 0.9994 & -0.0252 & 0.0222 & 0.0008 \\
Factor 3 & 0.0098 & 0.0257 & 0.9994 & -0.0230 & -0.0000 \\
Factor 4 & 0.1395 & -0.0226 & 0.0220 & 0.9894 & 0.0242 \\
Factor 5 & 0.0374 & -0.0004 & -0.0010 & -0.0297 & 0.9989 \\
\hline
\end{tabular}

Equamax

\begin{tabular}{lccccc}
\hline & Factor 1 & Factor 2 & Factor 3 & Factor 4 & Factor 5 \\
\hline Factor 1 & 0.9816 & -0.0051 & -0.0129 & -0.1680 & -0.0893 \\
Factor 2 & 0.0079 & 0.9993 & -0.0251 & 0.0228 & -0.0103 \\
Factor 3 & 0.0078 & 0.0256 & 0.9992 & -0.0275 & -0.0088 \\
Factor 4 & 0.1704 & -0.0229 & 0.0265 & 0.9846 & 0.0185 \\
Factor 5 & 0.0850 & 0.0105 & 0.0069 & -0.0334 & 0.9957 \\
\hline
\end{tabular}


Quartimax

\begin{tabular}{lccccc}
\hline & Factor 1 & Factor 2 & Factor 3 & Factor 4 & Factor 5 \\
\hline Factor 1 & 0.9911 & -0.0062 & -0.0129 & -0.1292 & -0.0295 \\
Factor 2 & 0.0089 & 0.9994 & -0.0253 & 0.0220 & 0.0034 \\
Factor 3 & 0.0104 & 0.0257 & 0.9994 & -0.0216 & 0.0021 \\
Factor 4 & 0.1300 & -0.0225 & 0.0206 & 0.9907 & 0.0264 \\
Factor 5 & 0.0257 & -0.0031 & -0.0029 & -0.0300 & 0.9992 \\
\hline
\end{tabular}

\begin{tabular}{lccccc}
\hline & Factor 1 & Factor 2 & Factor 3 & Factor 4 & factor 5 \\
\hline Factor 1 & 0.9815 & -0.0074 & -0.0139 & -0.1312 & -0.1384 \\
Factor 2 & 0.0054 & 0.9992 & -0.0253 & 0.0155 & -0.0272 \\
Factor 3 & 0.0077 & 0.0251 & 0.9991 & -0.0267 & -0.0220 \\
Factor 4 & 0.1342 & -0.0152 & 0.0261 & 0.9904 & 0.0106 \\
Factor 5 & 0.1361 & 0.0272 & 0.0192 & -0.0291 & 0.9897 \\
\hline
\end{tabular}

3.6.3. Information Criteria

\begin{tabular}{cc}
\hline Information Criteria & Values \\
\hline Log Likelihood & -230.0950 \\
Akaike & 470.1900 \\
Schwarz & 234.3424 \\
Hannan Quinne & 232.3969 \\
\hline
\end{tabular}

\subsection{For $n=70, p=10$ and $k=2$}

\subsubsection{Rotated Factor Loadings}

\begin{tabular}{ccccccccc}
\hline \multicolumn{2}{c}{ Varimax Loadings } & \multicolumn{2}{c}{ Equamax Loadings } & \multicolumn{2}{c}{ Quartimax Loadings } & \multicolumn{2}{c}{ Orthomax Loadings } \\
$\mathbf{I}$ & $\mathbf{I I}$ & $\mathbf{I}$ & $\mathbf{I I}$ & $\mathbf{I}$ & $\mathbf{I I}$ & I & II \\
\hline 1.0000 & -0.0057 & 1.0000 & -0.0048 & 1.0000 & -0.0059 & 1.0000 & -0.0037 \\
-0.0818 & -0.1573 & -0.0816 & -0.1574 & -0.0818 & -0.1573 & -0.0815 & -0.1575 \\
0.0385 & 0.1685 & 0.0383 & 0.1685 & 0.0385 & 0.1685 & 0.0381 & 0.1686 \\
-0.0663 & 0.0006 & -0.0663 & 0.0005 & -0.0663 & 0.0006 & -0.0663 & 0.0004 \\
-0.2759 & -0.1688 & -0.2757 & -0.1691 & -0.2759 & -0.1688 & -0.2755 & -0.1694 \\
-0.1151 & -0.0390 & -0.1151 & -0.0391 & -0.1152 & -0.0390 & -0.1151 & -0.0393 \\
-0.0401 & 0.0629 & -0.0401 & 0.0629 & -0.0401 & 0.0630 & -0.0402 & 0.0629 \\
0.1946 & 0.1182 & 0.1945 & 0.1184 & 0.1947 & 0.1182 & 0.1944 & 0.1186 \\
0.1780 & -0.0731 & 0.1780 & -0.0730 & 0.1779 & -0.0732 & 0.1781 & -0.0728 \\
-0.0041 & 1.0000 & -0.0050 & 1.0000 & -0.0038 & 1.0000 & -0.0061 & 1.0000 \\
\hline
\end{tabular}

\subsubsection{Factor Rotation Matrix}

Varimax

\begin{tabular}{ccc}
\hline & Factor I & Factor II \\
\hline Factor I & -0.0279 & 0.9996 \\
Factor II & 0.9996 & 0.0279 \\
\hline & Equamax \\
\hline & Factor I & Factor II \\
\hline Factor I & -0.0288 & 0.9996 \\
Factor II & 0.9996 & 0.0288 \\
\hline
\end{tabular}

Quartimax

\begin{tabular}{ccc}
\hline & Factor I & Factor II \\
\hline Factor I & -0.0277 & 0.9996 \\
Factor II & 0.9996 & 0.0277 \\
\hline & & \\
& Orthomax & \\
\hline & Factor I & Factor II \\
\hline Factor I & -0.0299 & 0.9996 \\
Factor II & 0.9996 & 0.0299 \\
\hline
\end{tabular}

\begin{tabular}{cc} 
3.7.3. Information Criteria & \\
\hline Information Criteria & Values \\
\hline Log Likelihood & -326.9945 \\
Akaike & 657.9890 \\
Schwarz & 328.8396 \\
Hannan Quinne & 328.0586 \\
\hline
\end{tabular}


3.8. For $n=70, p=10$, and $k=3$

3.8.1. Rotated Factor Loadings

\begin{tabular}{|c|c|c|c|c|c|c|c|c|c|c|c|}
\hline \multicolumn{3}{|c|}{ Varimax Loadings } & \multicolumn{3}{|c|}{ Equamax Loadings } & \multicolumn{3}{|c|}{ Quartimax Loadings } & \multicolumn{3}{|c|}{ Orthomax Loadings } \\
\hline $\begin{array}{c}\text { Factor } \\
\text { I }\end{array}$ & $\begin{array}{c}\text { Factor } \\
\text { II }\end{array}$ & $\begin{array}{c}\text { Factor } \\
\text { III }\end{array}$ & $\begin{array}{c}\text { Factor } \\
\text { I }\end{array}$ & $\begin{array}{c}\text { Factor } \\
\text { II }\end{array}$ & $\begin{array}{c}\text { Factor } \\
\text { III }\end{array}$ & $\begin{array}{c}\text { Factor } \\
\text { I }\end{array}$ & $\begin{array}{c}\text { Factor } \\
\text { II }\end{array}$ & $\begin{array}{c}\text { Factor } \\
\text { III }\end{array}$ & $\begin{array}{c}\text { Factor } \\
\text { I }\end{array}$ & $\begin{array}{c}\text { Factor } \\
\text { II }\end{array}$ & $\begin{array}{c}\text { Factor } \\
\text { III }\end{array}$ \\
\hline 0.9989 & -0.0199 & -0.0026 & 0.9998 & -0.0200 & 0.0047 & 0.9998 & -0.0198 & -0.0044 & 0.9996 & -0.0202 & 0.0176 \\
\hline-0.0792 & 0.1089 & -0.1916 & -0.0778 & 0.1101 & -0.1920 & -0.0796 & 0.1097 & -0.1915 & -0.0753 & 0.1087 & -0.1937 \\
\hline 0.0384 & 0.0163 & 0.1952 & 0.0369 & 0.1060 & 0.1955 & 0.0387 & 0.1064 & 0.1951 & 0.0344 & 0.1074 & 0.1959 \\
\hline-0.0707 & -0.2210 & 0.0189 & -0.0709 & -0.2210 & 0.0180 & -0.0707 & -0.2210 & 0.0191 & -0.0711 & -0.2209 & 0.0187 \\
\hline-0.2750 & 0.0242 & -0.2080 & -0.2735 & 0.0246 & -0.2100 & -0.2754 & 0.0241 & -0.2076 & -0.2708 & 0.0232 & -0.2137 \\
\hline-0.1164 & -0.0678 & -0.0494 & -0.1161 & -0.0677 & -0.0504 & -0.1165 & -0.0679 & -0.4092 & -0.1154 & -0.0681 & -0.0514 \\
\hline-0.0205 & 0.9995 & 0.0251 & -0.0205 & 0.9994 & 0.0266 & -0.0205 & 0.9995 & 0.0248 & -0.0206 & 0.9996 & 0.0109 \\
\hline 0.1947 & 0.0172 & 0.1319 & 0.1937 & 0.0169 & 0.1334 & 0.1949 & 0.0172 & 0.1316 & 0.1920 & 0.0178 & 0.1357 \\
\hline 0.1810 & 0.1431 & -0.1012 & 0.1818 & 0.1433 & -0.0997 & 0.1808 & 0.1431 & -0.1016 & 0.1830 & 0.1425 & -0.0983 \\
\hline-0.0067 & 0.0419 & 0.8396 & -0.0129 & 0.0405 & 0.8396 & -0.0052 & 0.0422 & 0.2933 & -0.0236 & 0.0465 & 0.8391 \\
\hline
\end{tabular}

3.8.2. Factor Rotation Matrix

\begin{tabular}{cccc}
\multicolumn{5}{c}{ Varimax } \\
\hline \multicolumn{5}{c}{ Factor I } & Factor II & Factor III \\
\hline Factor I & -0.179 & 0.9848 & 0.0251 \\
Factor II & 0.9851 & 0.1719 & 0.0022 \\
Factor III & 0.0021 & -0.0251 & 0.9997 \\
\hline \multicolumn{4}{c}{} \\
\hline \multicolumn{5}{c}{ Equamax } \\
\hline Factor I & Factor I & Factor II & Factor III \\
Factor II & -0.1720 & 0.9848 & 0.0254 \\
Factor III & 0.9851 & 0.1718 & 0.0097 \\
\hline & -0.0052 & -0.0267 & 0.9996 \\
\hline \multicolumn{4}{c}{} \\
\hline Quartimax \\
\hline Factor I & Factor I & Factor II & Factor III \\
Factor II & -0.1719 & 0.9848 & 0.0250 \\
Factor III & 0.9851 & 0.1720 & 0.0004 \\
\hline
\end{tabular}

Orthomax

\begin{tabular}{cccc}
\hline & Factor I & Factor II & Factor III \\
\hline Factor I & -0.1721 & 0.9850 & 0.0162 \\
Factor II & 0.9849 & 0.1717 & 0.0211 \\
Factor III & -0.0180 & -0.0196 & -0.9996 \\
\hline
\end{tabular}

\begin{tabular}{cc} 
3.8.3. Information Criteria & \\
\hline Information Criteria & Values \\
\hline Log Likelihood & -323.7360 \\
Akaike & 653.4720 \\
Schwarz & 326.5036 \\
Hannan Quinne & 325.3321 \\
\hline
\end{tabular}

\subsection{For $n=70, p=10$, and $k=5$}

\subsubsection{Rotated Factor Loadings}

\begin{tabular}{|c|c|c|c|c|c|c|c|c|c|}
\hline \multicolumn{5}{|c|}{ Varimax Loadings } & \multicolumn{5}{|c|}{ Equamax Loadings } \\
\hline 1 & 2 & 3 & 4 & 5 & 1 & 2 & 3 & 4 & 5 \\
\hline 0.9966 & -0.0598 & -0.0005 & 0.0554 & -0.0076 & 0.9949 & -0.0623 & 0.0128 & 0.0775 & -0.0141 \\
\hline-0.0639 & 0.0848 & -0.2353 & -0.1824 & 0.2870 & -0.0544 & 0.0854 & -0.2368 & -0.1856 & 0.2854 \\
\hline 0.0365 & -0.0451 & 0.2044 & -0.0216 & 0.0411 & 0.0346 & -0.0441 & 0.2050 & -0.0222 & 0.0405 \\
\hline-0.0659 & -0.0353 & -0.0008 & -0.0990 & -0.3618 & -0.0664 & -0.0346 & -0.0020 & -0.0967 & -0.3624 \\
\hline-0.2767 & -0.0161 & -0.2359 & 0.0071 & 0.0808 & -0.2732 & -0.0166 & -0.2393 & 0.0006 & 0.0830 \\
\hline-0.0573 & 0.9977 & -0.0142 & 0.0324 & -0.0085 & -0.0559 & 0.9975 & -0.0193 & 0.0387 & -0.0082 \\
\hline-0.0487 & -0.0687 & 0.0752 & 0.1504 & 0.5611 & -0.0490 & -0.0690 & 0.0758 & 0.1423 & 0.5631 \\
\hline 0.2154 & 0.0999 & 0.1252 & -0.2546 & 0.0801 & 0.2203 & 0.1019 & 0.1268 & -0.2502 & 0.0755 \\
\hline 0.1457 & 0.0920 & -0.0539 & 0.7086 & 0.0813 & 0.1312 & 0.0862 & -0.0501 & 0.7116 & 0.0882 \\
\hline-0.0080 & -0.0265 & 0.7554 & -0.0507 & 0.0269 & -0.0164 & -0.0227 & 0.7552 & -0.0540 & 0.0258 \\
\hline \multicolumn{5}{|c|}{ Quartimax Loadings } & \multicolumn{5}{|c|}{ Orthomax Loadings } \\
\hline 1 & 2 & 3 & 4 & 5 & 1 & 2 & 3 & 4 & 5 \\
\hline 0.9969 & -0.0592 & -0.0036 & 0.0504 & -0.0063 & 0.9943 & -0.0633 & 0.0358 & 0.0766 & -0.0165 \\
\hline-0.0660 & 0.0847 & -0.2348 & -0.1817 & 0.2878 & -0.0484 & 0.0799 & -0.2312 & -0.1917 & 0.2887 \\
\hline
\end{tabular}




\begin{tabular}{cccccccccc}
\hline \multicolumn{3}{c}{ Varimax Loadings } & $\mathbf{5}$ & \multicolumn{3}{c}{ Equamax Loadings } \\
$\mathbf{1}$ & $\mathbf{2}$ & $\mathbf{3}$ & $\mathbf{4}$ & $\mathbf{5}$ & $\mathbf{1}$ & $\mathbf{2}$ & $\mathbf{3}$ & $\mathbf{4}$ & $\mathbf{5}$ \\
\hline 0.0370 & 0.0454 & 0.2043 & -0.0214 & 0.0412 & 0.0300 & -0.0447 & 0.2066 & -0.0267 & 0.0317 \\
-0.0659 & -0.0355 & -0.0005 & -0.0996 & -0.3616 & -0.0677 & -0.0317 & -0.0182 & -0.0839 & -0.3651 \\
-0.2775 & -0.0160 & -0.2351 & 0.0085 & 0.0804 & -0.2675 & -0.0171 & -0.2421 & 0.0028 & 0.0925 \\
-0.0577 & 0.9977 & -0.0129 & 0.0308 & -0.0085 & -0.0533 & 0.9980 & -0.0207 & 0.0266 & 0.0045 \\
-0.0485 & -0.0686 & 0.0751 & 0.1524 & 0.5606 & -0.489 & -0.0732 & 0.0974 & 0.1230 & 0.5637 \\
0.2143 & 0.0994 & 0.1249 & -0.2555 & 0.0811 & 0.2174 & 0.0976 & 0.1294 & -0.2566 & 0.0628 \\
0.1490 & 0.0934 & -0.0549 & 0.7079 & 0.0795 & 0.1338 & 0.0938 & -0.0375 & 0.7077 & 0.1137 \\
-0.0060 & -0.0274 & 0.7554 & -0.0499 & 0.0271 & -0.0332 & -0.0230 & 0.7539 & -0.0680 & -0.0034 \\
\hline
\end{tabular}

\subsubsection{Factor Rotation Matrix}

Varimax.

\begin{tabular}{lllccc}
\hline & Factor 1 & Factor 2 & Factor 3 & Factor 4 & Factor 5 \\
\hline Factor 1 & 0.9966 & -0.0907 & -0.0000 & 0.0542 & -0.0073 \\
Factor 2 & 0.0885 & 0.9951 & -0.0143 & 0.0407 & -0.0096 \\
Factor 3 & 0.0236 & 0.0266 & 0.9130 & -0.3987 & -0.0788 \\
Factor 4 & -0.0446 & -0.0208 & 0.3988 & 0.8350 & 0.3759 \\
Factor 5 & 0.0290 & 0.0204 & -0.0846 & -0.3731 & 0.9232 \\
\hline
\end{tabular}

Equamax

\begin{tabular}{llllll}
\hline & Factor 1 & Factor 2 & Factor 3 & Factor 4 & Factor 5 \\
\hline Factor 1 & 0.9926 & -0.0932 & 0.0133 & 0.0759 & -0.0138 \\
Factor 2 & 0.0896 & 0.9945 & -0.0175 & 0.0503 & -0.0104 \\
Factor 3 & 0.0204 & 0.0336 & 0.9118 & -0.4000 & -0.0842 \\
Factor 4 & -0.0657 & -0.0249 & 0.4012 & 0.8280 & 0.3853 \\
Factor 5 & 0.0453 & 0.0233 & -0.0848 & -0.3822 & 0.9188 \\
\hline
\end{tabular}

Quartimax

\begin{tabular}{llllll}
\hline & Factor 1 & Factor 2 & Factor 3 & Factor 4 & Factor 5 \\
\hline Factor 1 & 0.9947 & -0.0901 & -0.0032 & 0.0493 & -0.0060 \\
Factor 2 & 0.0882 & 0.9952 & -0.0135 & 0.0384 & -0.0095 \\
Factor 3 & 0.0244 & 0.0249 & 0.9133 & -0.3982 & -0.0776 \\
Factor 4 & -0.0397 & -0.0198 & 0.3981 & 0.8367 & 0.3735 \\
Factor 5 & 0.0255 & 0.0197 & -0.0844 & -0.3708 & 0.9243 \\
\hline
\end{tabular}

Orthomax

\begin{tabular}{llllll}
\hline & Factor 1 & Factor 2 & Factor 3 & Factor 4 & Factor 5 \\
\hline Factor 1 & 0.9919 & -0.0942 & 0.0363 & 0.0754 & -0.0166 \\
Factor 2 & 0.0922 & 0.9949 & -0.0155 & 0.0380 & 0.0021 \\
Factor 3 & -0.0008 & 0.0302 & 0.9006 & -0.4137 & -0.1298 \\
Factor 4 & -0.0722 & -0.0183 & 0.4289 & 0.8078 & 0.3975 \\
Factor 5 & 0.494 & 0.0083 & -0.0584 & -0.4114 & 0.9082 \\
\hline
\end{tabular}

\subsubsection{Information Criteria}

\begin{tabular}{cc}
\hline Information Criteria & Values \\
\hline Log Likelihood & -319.8686 \\
Akaike & 649.7370 \\
Schwarz & 324.4814 \\
Hannan Quinne & 322.5287 \\
\hline
\end{tabular}

\section{Discussion of Results and Conclusion}

When the sample size (n) is 30 and the number of variables (p) is 10, the values of the Akaike (AIC) and Schwarz (SIC) Information criteria for different number of retained factors are as follows: for $\mathrm{k}=2, \mathrm{AIC}, \mathrm{SIC}$ and
HQIC values are 237.2530, 118.1036, and 117.304 respectively; for $\mathrm{k}=3 \mathrm{AIC}$ is 231.2490 , SIC is 114.8402 and HQIC is 109.9072 ; for $\mathrm{k}=5$, AIC is 226.4260 , SIC is 111.9058 and HQIC is 109.9072 .

On increasing the sample size to 50 and retaining the same number of variables i.e. 10 ; for $\mathrm{k}=2$, AIC is 479.6350 , SIC is 239.6635 and HQIC is 238,7380 ; for $\mathrm{k}=3$, AIC is 474.2300, SIC is 236.6635 and HQIC is 235.4961 and finally if $\mathrm{k}=5$, AIC is 470.1910 , SIC is 234.3424 and HQIC is 232.3969 .

Finally, when the sample size is increased to 70 and the same number of variables maintained, for $\mathrm{k}=2$, AIC is 657.9890, SIC is 328.8396 and HQIC is 328.0586 ; for $\mathrm{k}=$ 3 , AIC is 653.4720 . SIC is 326.5036 and HQIC is 325.3321 ; then for $\mathrm{k}=5$, AIC is 649.7370 , SIC is 324.4814 and HQIC is 322.5287 .

The factor rotation matrix for all the sample sizes and the number of parameters retained as considered is almost the same for all the four methods of rotation considered here.

In conclusion based on the results above, it shows that the values for both AIC, SIC and HQIC all decreases for all the sample sizes considered as k increases. Again, as we moved from on sample size to the other, the values of the AIC, SIC and HQIC increases as the sample size increases for all the number of retained factors considered. Also, since in the information criteria model selection, the model with the least values of AIC, SIC and HQIC is considered as the best model. Here, the AIC, the SIC and the HQIC values are least for $\quad k=5$ for all the sample sizes considered. Hence, the optimal number of factors to using the Maximum Likelihood Estimation method based on the simulated data used is five (5).

\section{References}

[1] Akaike, H. (1973). Information Theory and Extension of the Maximum Likelihood Principle; Second International Symposium on Information Theory (B.N. Petrov and F. Csaki, Eds.). Budapest Hungary: Akademia Kiado, 267-281

[2] Comrey, A.L., and Lee, H.B. (1992). A First Course in Factor Analysis (2 ${ }^{\text {nd }}$ Ed.). Hillsdale, NJ: Erlbaum.

[3] Harman, H.H. (1976). Modern Factor Analysis (3 ${ }^{\text {rd }}$ Ed.). Chicago: The University of Chicago Press.

[4] Johnson, R.A., and Wichern, D.W. (2007). Applied Multivariate Statistical Analysis ( $6^{\text {th }}$ Ed.). Prentics Hall, Englewood Cliffs, New Jersey. 
[5] Onyeagu, S.I. (2003). A First Course in Multivariate Statistical Analysis ( $1^{\text {st }}$ Ed.). Mega Concept Publishers.
[6] Schwarz,G.(1978). Estimating the Dimension of a Model. Annals of Statistics 6, 461-464. 